This item was submitted to Loughborough's Research Repository by the author.

Items in Figshare are protected by copyright, with all rights reserved, unless otherwise indicated.

\title{
A novel method for the partition of mixed-mode fractures in 2D elastic laminated unidirectional composite beams
}

PLEASE CITE THE PUBLISHED VERSION

http://dx.doi.org/10.1016/j.compstruct.2014.05.041

PUBLISHER

(C) Elsevier

VERSION

AM (Accepted Manuscript)

LICENCE

CC BY-NC-ND 4.0

\section{REPOSITORY RECORD}

Harvey, Christopher, Joe Wood, Simon Wang, and Andrew Watson. 2014. "A Novel Method for the Partition of Mixed-mode Fractures in 2D Elastic Laminated Unidirectional Composite Beams". Loughborough University. https://hdl.handle.net/2134/13836. 
This item was submitted to Loughborough's Institutional Repository (https://dspace.lboro.ac.uk/) by the author and is made available under the following Creative Commons Licence conditions.

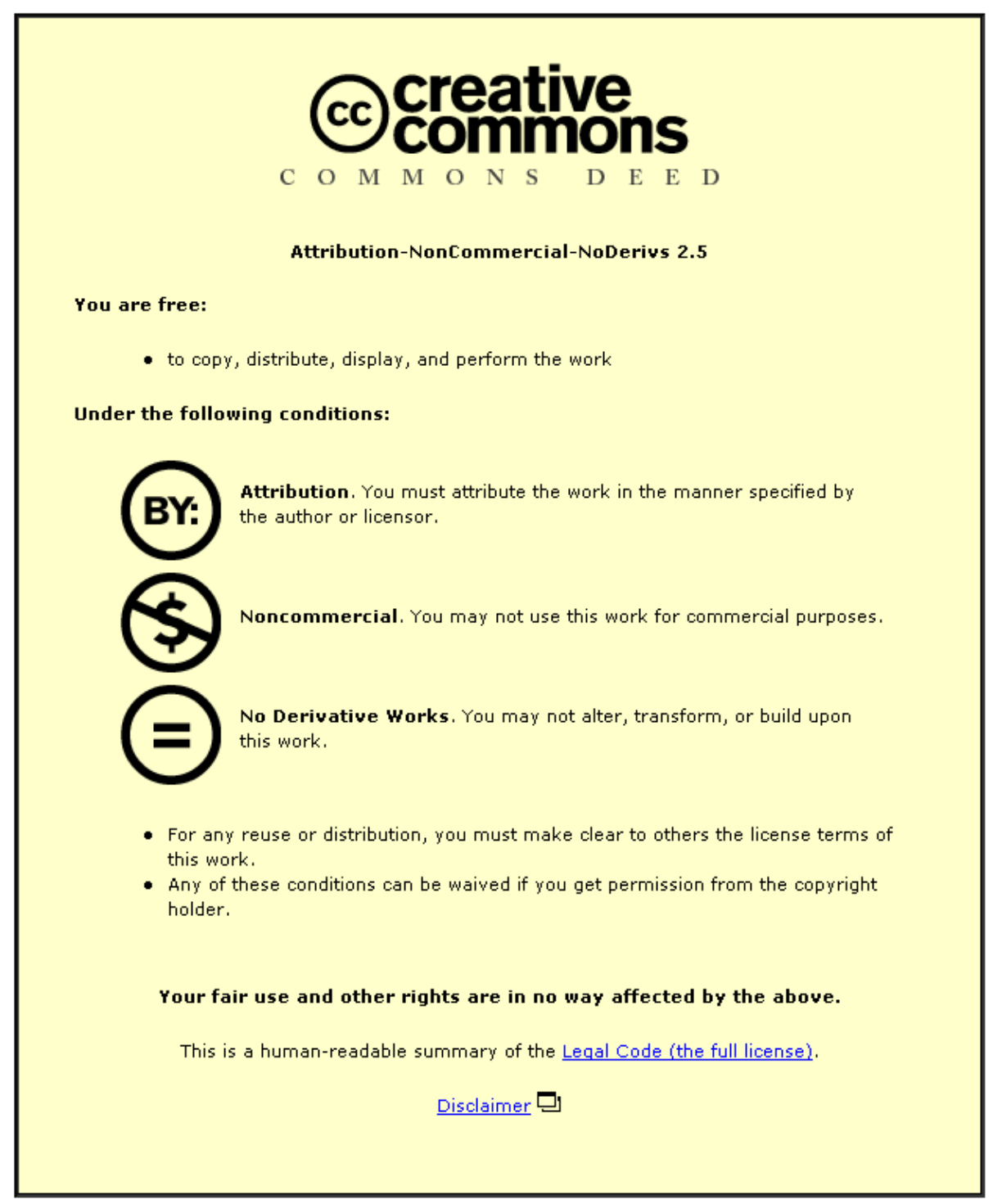

For the full text of this licence, please go to: http://creativecommons.org/licenses/by-nc-nd/2.5/ 


\title{
A novel method for the partition of mixed-mode fractures in 2D elastic laminated unidirectional composite beams
}

\author{
C. M. Harvey, J. D. Wood, S. Wang*, A. Watson \\ Department of Aeronautical and Automotive Engineering, Loughborough University, \\ Loughborough, Leicestershire LE11 3TU, United Kingdom
}

\begin{abstract}
A powerful method for partitioning mixed-mode fractures on rigid interfaces in laminated unidirectional double cantilever beams (DCBs) is developed by taking 2D elasticity into consideration in a novel way. Pure modes based on 2D elasticity are obtained by introducing correction factors into the beam-theory-based mechanical conditions. These 2D-elasticity-based pure modes are then used to derive a 2D-elasticity-based partition theory for mixed-mode fractures. Excellent agreement is observed between the present partition theory and Suo and Hutchinson's partition theory [1]. Furthermore, the method that is developed in this work has a stronger capability for solving more complex mixed-mode partition problems, for example, in the bimaterial case.
\end{abstract}

[1] Suo Z, Hutchinson JW. Interface crack between two elastic layers. International Journal of Fracture Mechanics 1990;43:1-18.

Keywords: Energy release rate, Fracture mode partitioning, Laminated unidirectional composites, Mixed-mode fracture, Orthogonal pure modes

\section{Introduction}

The present work revisits the partitioning of mixed-mode fractures in laminated unidirectional composite double cantilever beams (DCBs) with rigid interfaces by taking 2D elasticity into consideration in a novel way. In Suo and Hutchinson's work [1], conventional 2D elasticity theory is employed in conjunction with stress intensity factors in order to give accurate partitions. This conventional approach however often has limitations in dealing with more complex

\footnotetext{
* Corresponding Author

Email addresses:

S.wang@lboro.ac.uk (S. Wang), c.m. harvey@lboro.ac.uk (C. M. Harvey),

J.Wood@lboro.ac.uk (J. D. Wood), A.Watson@lboro.ac.uk (A. Watson)
} 


\begin{tabular}{|c|c|}
\hline \multicolumn{2}{|c|}{ Nomenclature } \\
\hline$a$ & crack length in a DCB \\
\hline$A_{1}, A_{2}, A$ & cross section areas of upper, lower and intact beams \\
\hline$b$ & width of a DCB \\
\hline$c_{\theta}, c_{\beta}$ & 2D elasticity correction factors for $\theta$ mode I and $\beta$ mode II \\
\hline$E$ & Young's modulus \\
\hline$G, G_{I}, G_{I I}$ & total, mode I and II ERRs \\
\hline$G_{\theta}, G_{\beta}$ & $\theta$ mode I and $\beta$ mode II ERRs \\
\hline$h_{1}, h_{2}, h$ & thicknesses of upper, lower and intact beams \\
\hline$I_{1}, I_{2}, I$ & second moments of upper, lower and intact beams \\
\hline$L$ & length of a DCB \\
\hline$M_{1}, M_{2}$ & DCB tip bending moments on upper and lower beams \\
\hline$M_{1 B}, M_{2 B}$ & crack tip bending moments on upper and lower beams \\
\hline$N_{1}, N_{2}$ & DCB tip axial forces on upper and lower beams \\
\hline$N_{1 B}, N_{2 B}$ & crack tip axial forces on upper and lower beams \\
\hline$N_{1 B e}$ & crack tip effective axial force on upper beam \\
\hline$\gamma$ & thickness ratio \\
\hline$(\theta, \beta)$ & zero shearing displacement, zero opening force orthogonal pure modes pair \\
\hline$\left(\theta^{\prime}, \beta^{\prime}\right)$ & zero shearing force, zero opening displacement orthogonal pure modes pair \\
\hline$v$ & Poisson’s ratio \\
\hline$\sigma_{n}, \tau_{s}$ & interface normal and shear stresses \\
\hline$\Delta a$ & crack influence length in a DCB \\
\hline \multicolumn{2}{|c|}{ Abbreviations } \\
\hline DCB & double cantilever beam \\
\hline ERR & energy release rate \\
\hline
\end{tabular}

problems, for example, in the bimaterial case where the partition relies on extensively tabulated numerical results over a finite range of geometries and material configurations [1]. The present 
work aims to develop a novel and powerful method to calculate energy release rate (ERR) partitions with the same level of accuracy as in Suo and Hutchinson's work [1]. Furthermore, it aims for the method to have a stronger capability for solving more complex mixed-mode partition problems (like the bimaterial one described above) than the conventional method in Ref. [1] has. The structure of the paper is as follows. The novel method is developed in Section 2. Comparisons with several existing partition theories are presented in Section 3. In particular, these comparisons include ones against Suo and Hutchinson's partition theory [1] since it is regarded as the most accurate. Conclusions are made in Section 4.

\section{Development of the novel method}

Fig. 1a shows a laminated unidirectional composite DCB with its geometry and tip bending moments $M_{1}$ and $M_{2}$, and axial forces $N_{1}$ and $N_{2}$. The crack influence zone extends to a point A, a $\Delta a$-distance ahead of the crack tip B. Fig. 1b only shows the sign convention of the interface normal stress $\sigma_{n}$ and shear stress $\tau_{s}$ instead of any representative distribution. Beyond point A, both the normal stress $\sigma_{n}$ and shear stress $\tau_{s}$ becomes zero.

Based on the authors' previous work [2-5], the total ERR $G$ is calculated as follows:

$$
\begin{aligned}
& G=\frac{1}{2 \bar{E} b}\left[\frac{M_{1 B}^{2}}{I_{1}}+\frac{M_{2 B}^{2}}{I_{2}}-\frac{1}{I}\left(M_{1 B}+M_{2 B}-\frac{h_{2} N_{1 B e}}{2}\right)^{2}+\left(\frac{1}{A_{1}}-\frac{1}{A}\right) N_{1 B e}^{2}\right] \\
& =\left\{\begin{array}{lll}
M_{1 B} & M_{2 B} & N_{1 B e}
\end{array}\right\}[C]\left\{\begin{array}{lll}
M_{1 B} & M_{2 B} & N_{1 B e}
\end{array}\right\}^{T}
\end{aligned}
$$

where $N_{1 B e}=N_{1 B}-N_{2 B} / \gamma$ with $\gamma=h_{2} / h_{1}, b$ is the width of the beam, and $\bar{E}$ is the effective axial Young's modulus for orthotropic material; for isotropic material then $\bar{E}=E /\left(1-\nu^{2}\right)$ for plane strain and $\bar{E}=E$ for plane stress where $E$ is the Young's modulus and $\nu$ the Poisson's ratio [1]. $M_{1 B}$ and $M_{2 B}$ are the two bending moments at the crack tip B, and $N_{1 B}$ and $N_{2 B}$ are the axial forces at the crack tip B. Other symbols have their conventional meanings. $G$ is of quadratic form in terms of $M_{1 B}, M_{2 B}$ and $N_{1 B e}$ with the coefficient matrix [C], which is given in full in the Appendix. The total ERR is the same for both the Euler and Timoshenko beam theories and for 2D elasticity theory. The mode I and II partitions of ERR are however different. Approximate 2D partition theories have been given in Refs. [2-4]. Here, a partition theory of the same level of accuracy as that of the work in Ref. [1] is obtained by developing a novel and powerful method. 
By using the same hypothesis as in Refs. [2-4,6], namely that there generally exist two sets of orthogonal pure modes for rigid interface fracture in DCBs, the total ERR $G$ in Eq. (1) can be partitioned as

$$
\begin{gathered}
G_{I}=c_{I}\left(M_{1 B}-\frac{M_{2 B}}{\beta_{1-2 D}}-\frac{N_{1 B e}}{\beta_{2-2 D}}\right)\left(M_{1 B}-\frac{M_{2 B}}{\beta_{1-2 D}^{\prime}}-\frac{N_{1 B e}}{\beta_{2-2 D}^{\prime}}\right) \\
G_{I I}=c_{I I}\left(M_{1 B}-\frac{M_{2 B}}{\theta_{1-2 D}}-\frac{N_{1 B e}}{\theta_{2-2 D}}\right)\left(M_{1 B}-\frac{M_{2 B}}{\theta_{1-2 D}^{\prime}}-\frac{N_{1 B e}}{\theta_{2-2 D}^{\prime}}\right)
\end{gathered}
$$

where $c_{I}$ and $c_{I I}$ are two constants, and $\left(\theta_{i-2 D}, \beta_{i-2 D}\right)$ and $\left(\theta_{i-2 D}^{\prime}, \beta_{i-2 D}^{\prime}\right)$ with $i=1,2$ represent the first and second sets orthogonal pure modes respectively. The subscript $2 D$ denotes that the pure modes are based on 2D elasticity theory. For example, when $M_{2 B}=\theta_{1-2 D} M_{1 B}$ and $N_{1 B e}=0$, the pure mode I mode occurs as the relative shearing displacement just behind the crack tip is zero. This pure mode I is denoted by $\theta_{1-2 D}$. Its orthogonal pure mode II is $\beta_{1-2 D}$ which corresponds to zero crack tip opening force. Here, the 'orthogonal' means

$$
\left\{\begin{array}{lll}
1 & \theta_{1-2 D} & 0
\end{array}\right\}[C]\left\{11 \quad \beta_{1-2 D} \quad 0\right\}^{T}=0
$$

For simplicity, Eq. (4) can be written as $\theta_{1-2 D}=\operatorname{orthogonal}\left(\beta_{1-2 D}\right)$. Similarly, when $M_{2 B}=\theta_{1-2 D}^{\prime} M_{1 B}$ and $N_{1 B e}=0$, the pure mode I mode occurs as the crack tip shearing force is zero. This pure mode $\mathrm{I}$ is denoted by $\theta_{1-2 D}^{\prime}$. Its orthogonal pure mode II is $\beta_{1-2 D}^{\prime}$ which corresponds to zero crack tip opening displacement.

The work in Refs. [2-4,6] has shown that in Euler beam theory with rigid interfaces, the two sets of orthogonal pure modes do not coincide and that this results in 'stealthy' interactions which change the ERR partitions $G_{I}$ and $G_{I I}$ but do not change the total ERR $G$. The work in Ref. [2-6] also shows that in Timoshenko beam theory with either rigid or non-rigid interfaces, these two sets of modes coincide on the first set of pure modes from Euler beam theory resulting in no stealthy interaction. Furthermore, Ref. [1] shows that the two sets also coincide in 2D elasticity theory for rigid interfaces, i.e. $\left(\theta_{i-2 D}, \beta_{i-2 D}\right)=\left(\theta_{i-2 D}^{\prime}, \beta_{i-2 D}^{\prime}\right)$ with $i=1,2$. Therefore, Eqs (2) and (3) become here for laminated unidirectional composite DCBs with rigid interfaces in 2D elasticity,

$$
G_{I}=c_{I}\left(M_{1 B}-\frac{M_{2 B}}{\beta_{1-2 D}}-\frac{N_{1 B e}}{\beta_{2-2 D}}\right)^{2}
$$




$$
G_{I I}=C_{I I}\left(M_{1 B}-\frac{M_{2 B}}{\theta_{1-2 D}}-\frac{N_{1 B e}}{\theta_{2-2 D}}\right)^{2}
$$

where

$$
\begin{gathered}
c_{I}=G_{\theta_{1-2 D}}\left(1-\frac{\theta_{1-2 D}}{\beta_{1-2 D}}\right)^{-2}, \quad c_{I I}=G_{\beta_{1-2 D}}\left(1-\frac{\beta_{1-2 D}}{\theta_{1-2 D}}\right)^{-2} \\
G_{\theta_{1-2 D}}=\frac{1}{2 b E}\left(\frac{1}{I_{1}}+\frac{\theta_{1-2 D}^{2}}{I_{2}}-\frac{\left(1+\theta_{1-2 D}\right)^{2}}{I}\right), \quad G_{\beta_{1-2 D}}=\frac{1}{2 b E}\left(\frac{1}{I_{1}}+\frac{\beta_{1-2 D}^{2}}{I_{2}}-\frac{\left(1+\beta_{1-2 D}\right)^{2}}{I}\right)
\end{gathered}
$$

Now, the key task is to determine the orthogonal pure mode set $\left(\theta_{i-2 D}, \beta_{i-2 D}\right)$ with $i=1,2$. At this point, it is important to note that the orthogonal property demonstrated in Eq. (4) exists between any pair of pure modes in the pure mode set $\left(\theta_{i-2 D}, \beta_{i-2 D}\right)$ with $i=1,2$. That is, $\theta_{1-2 D}=\operatorname{orthogonal}\left(\beta_{1-2 D}\right.$ and $\left.\beta_{2-2 D}\right)$ and $\theta_{2-2 D}=\operatorname{orthogonal}\left(\beta_{1-2 D}\right.$ and $\left.\beta_{2-2 D}\right)$. As long as one pure mode is known, say $\theta_{1-2 D}$, the others can be obtained by using the orthogonal property. This knowledge provides a powerful methodology to find all the pure modes and to partition mixed modes, which will be used in the following development. It is seen now that the central task of the present work is to determine $\theta_{1-2 D}$. In what follows, a novel method is developed for this task.

To achieve this task only the simplest loading case is required: a DCB with two crack tip bending moments $M_{1 B}$ and $M_{2 B}$. When Euler beam theory is used, there are two distinct sets of orthogonal pure modes [2-4,6]: In the first set of pure modes, the pure mode I mode is given by $M_{2 B}=\theta_{1} M_{1 B}=-\left(h_{2} / h_{1}\right)^{2} M_{1 B}=-\gamma^{2} M_{1 B} \quad$, and the pure mode II mode by $M_{2 B}=\beta_{1} M_{1 B}=\gamma^{2}(3+\gamma) /(1+3 \gamma) M_{1 B}$. In the second set of pure modes, the pure mode I mode is given by $M_{2 B}=\theta_{1}^{\prime} M_{1 B}=-M_{1 B}$, and pure mode II mode by $M_{2 B}=\beta_{1}^{\prime} M_{1 B}=\gamma^{3} M_{1 B}$. When Timoshenko beam theory is used, the two sets of pure modes coincide on the first set. That is, $\left(\theta_{1-T}, \beta_{1-T}\right)=\left(\theta_{1-T}^{\prime}, \beta_{1-T}^{\prime}\right)=\left(\theta_{1}, \beta_{1}\right)$ where the subscript $T$ denotes for Timoshenko beam theory. This is due to the introduction of the drastic uniform through-thickness shearing strain by the Timoshenko beam theory. It is therefore reasonable to anticipate that the pure mode set $\left(\theta_{1-2 D}, \beta_{1-2 D}\right)$ in $2 \mathrm{D}$ elasticity theory will be bounded by the $\left(\theta_{1}, \beta_{1}\right)$ set and the $\left(\theta_{1}^{\prime}, \beta_{1}^{\prime}\right)$ set because $2 \mathrm{D}$ elasticity theory more appropriately considers the mechanics at the crack tip. That is, 
$\theta_{1-2 D}$ is between $\theta_{1}$ and $\theta_{1}^{\prime}$, and $\beta_{1-2 D}$ is between $\beta_{1}$ and $\beta_{1}^{\prime}$. To determine $\theta_{1-2 D}$ and $\beta_{1-2 D}$, the beam-theory-based mechanical conditions for the $\theta_{1}$ and $\beta_{1}$ pure modes are re-examined.

The resultant moment $M_{n}$ about point A due to $\sigma_{n}$ within the $\Delta a$ region is given by [3,4]

$$
M_{n}=b \int_{0}^{\Delta a} \int_{0}^{x} \sigma_{n} d x d x=b \int_{0}^{\Delta a} \sigma_{n}(\Delta a-x) d x=M_{n m}+F_{n} \Delta a
$$

where $M_{n m}=-b \int_{0}^{\Delta a} x \sigma_{n} d x$ and $F_{n}=-b \int_{0}^{\Delta a} \sigma_{n} d x$. Note that physically $M_{n m}$ is the resultant moment about the crack tip at point B due to $\sigma_{n}$, and that $F_{n}$ is the resultant normal force due to $\sigma_{n}$, which is zero since there are no crack tip shear forces. At point A, there is a continuity of curvature condition. By considering this condition, $M_{n m}$ is calculated to be

$$
M_{n m}=\frac{1}{h_{1}+h_{2}}\left\{h_{2}\left[M_{1 B}-\frac{I_{1}}{I}\left(M_{1 B}+M_{2 B}\right)\right]-h_{1}\left[M_{2 B}-\frac{I_{2}}{I}\left(M_{1 B}+M_{2 B}\right)\right]\right\}
$$

It is important to note that Eqs. (9) and (10) remain the same regardless of whether Euler or Timoshenko beam theory or 2D elasticity theory is used because no deformation is considered within the $\Delta a$ region. In both the Euler and Timoshenko beam theories, the pure mode II condition $M_{2 B}=\beta_{1} M_{1 B}$ is obtained by setting $M_{n m}=0$, which produces

$$
\frac{M_{1 B}}{I_{1}}-\frac{M_{1 B}+M_{2 B}}{I}=\gamma^{2}\left(\frac{M_{2 B}}{I_{2}}-\frac{M_{1 B}+M_{2 B}}{I}\right)
$$

It can be shown that the physical meaning of this condition in the Euler and Timoshenko beam theories is uniformly zero normal stress within the $\Delta a$ region. Obviously, in 2D elasticity theory, this condition does not produce the same stress distribution and therefore also does not represent the pure mode condition. Therefore the condition in Eq. (11) needs to be corrected before it can apply to 2D elasticity theory. Note that the left side of Eq. (11) gives the difference between the curvature at the crack tip and the curvature at point A for the upper beam, which is related to the difference between the curvature at the crack tip and the curvature at point $\mathrm{A}$ for the lower beam by the right side of Eq. (11). It is expected that these differences between the curvatures will be different in 2D elasticity theory; hence, a correction factor $c_{\beta}(\gamma)$ is introduced in Eq. (11) as follows:

$$
\frac{M_{1 B}}{I_{1}}-c_{\beta} \frac{M_{1 B}+M_{2 B}}{I}=\gamma^{2}\left(\frac{M_{2 B}}{I_{2}}-c_{\beta} \frac{M_{1 B}+M_{2 B}}{I}\right)
$$


This condition gives the pure mode II mode in 2D elasticity as

$$
\beta_{1-2 D}=\gamma\left[\frac{(1+\gamma)^{2}+c_{\beta}(\gamma-1)}{(1+\gamma)^{2}-c_{\beta} \gamma(\gamma-1)}\right]
$$

It is worth noting from Eqs. (12) and (13) that when $\gamma=1, \beta_{1-2 D}=\beta_{1}=\beta_{1}^{\prime}=1$ and $c_{\beta}(1)$ can take any value. However, when $\gamma \neq 1$, it is reasonable to assume that $\beta_{1-2 D}$ is bounded by $\beta_{1}$ and $\beta_{1}^{\prime}$, i.e. $\beta_{1}<\beta_{1-2 D}<\beta_{1}^{\prime}$ when $\gamma>1$, and $\beta_{1}^{\prime}<\beta_{1-2 D}<\beta_{1}$ when $\gamma<1$, as mentioned earlier. The bounds of the correction factor $c_{\beta}(\gamma)$ are therefore found to be

$$
1<c_{\beta}(\gamma)<\left[\hat{c}_{\beta}(\gamma)=\frac{(1+\gamma)^{3}}{1+\gamma^{3}}\right]
$$

Note that $c_{\beta}(\gamma)=c_{\beta}(1 / \gamma)$ because $\beta_{1-2 D}(\gamma)=1 / \beta_{1-2 D}(1 / \gamma)$ due to mechanical symmetry.

In both the Euler and Timoshenko beam theories, the pure mode I condition $M_{2 B}=\theta_{1} M_{1 B}$ is obtained by zeroing the relative shearing displacement at the crack tip, i.e.

$$
\frac{M_{1 B}}{I_{1}}=-\gamma\left(\frac{M_{2 B}}{I_{2}}\right)
$$

Similarly, a correction factor $c_{\theta}(\gamma)$ is introduced in Eq. (15) as follows:

$$
\frac{M_{1 B}}{I_{1}}+c_{\theta}\left(\frac{M_{1 B}+M_{2 B}}{I}\right)=-\gamma\left[\frac{M_{2 B}}{I_{2}}+c_{\theta}\left(\frac{M_{1 B}+M_{2 B}}{I}\right)\right]
$$

This condition gives the pure mode I mode in 2D elasticity as

$$
\theta_{1-2 D}=-\gamma^{2}\left[\frac{(1+\gamma)^{2}+c_{\theta}}{(1+\gamma)^{2}+c_{\theta} \gamma^{2}}\right]
$$

Similarly, it is worth noting from Eq. (17) that when $\gamma=1, \theta_{1-2 D}=\theta_{1}=\theta_{1}^{\prime}=-1$ and $c_{\theta}(1)$ can take any value. However when $\gamma \neq 1$, it is reasonable to assume that $\theta_{1-2 D}$ is bounded by $\theta_{1}$ and $\theta_{1}^{\prime}$, i.e. $\theta_{1}<\theta_{1-2 D}<\theta_{1}^{\prime}$ when $\gamma>1$, and $\theta_{1}^{\prime}<\theta_{1-2 D}<\theta_{1}$ when $\gamma<1$, as mentioned earlier. The bounds of the correction factor $c_{\theta}(\gamma)$ are therefore found to be

$$
0<c_{\theta}(\gamma)<\left[\hat{c}_{\theta}(\gamma)=\infty\right]
$$

Also note that $c_{\theta}(\gamma)=c_{\theta}(1 / \gamma)$ because $\theta_{1-2 D}(\gamma)=1 / \theta_{1-2 D}(1 / \gamma)$ due to mechanical symmetry. 
The relationship between the two correction factors $c_{\theta}(\gamma)$ and $c_{\beta}(\gamma)$ can be found by using the orthogonal property between the pure modes $\theta_{1-2 D}$ and $\beta_{1-2 D}[2-6]$. The relationships are

$$
c_{\theta}(\gamma)=\frac{\left(1-c_{\beta}\right)(1+\gamma)^{3}}{\left(1+\gamma^{3}\right) c_{\beta}-(1+\gamma)^{3}}
$$

and

$$
c_{\beta}(\gamma)=\frac{\left(1+c_{\theta}\right)(1+\gamma)^{3}}{\left(1+\gamma^{3}\right) c_{\theta}+(1+\gamma)^{3}}
$$

At this point, it is helpful to pay attention to the values of $c_{\theta}(1)$ and $c_{\beta}(1)$. It is seen from Eq. (16) that the two correction terms represent the contributions of the curvature of the intact beam to those of the upper and lower beams at the crack tip. Therefore for $\gamma=1$, it is reasonable to assume that the corrected curvatures are those obtained by averaging those of the intact beam and two separated beams. This argument gives $c_{\theta}(1)=1$ which then gives $c_{\beta}(1)=1.6$ from Eq. (20). It is worth noting that the work in Ref. [7] effectively uses the constant $c_{\theta}=1$.

It is seen from Eq. (20) that when $\gamma$ tends to infinity or to zero, the correction factor $c_{\beta}(\gamma)$ tends to a constant unit value, $\bar{c}_{\beta}=1$. Similarly, it is expected that when $\gamma$ tends to infinity or to zero, the correction factor $c_{\theta}(\gamma)$ also tends to a constant value, $\bar{c}_{\theta}$. This $\bar{c}_{\theta}$ value needs to be determined. When $\gamma$ tends to infinity or zero, Eq. (20) gives

$$
\lim _{\gamma \rightarrow \infty}\left\{\frac{d c_{\beta}(\gamma)}{d \gamma}\right\}=0 \text { and } \lim _{\gamma \rightarrow 0}\left\{\frac{d c_{\beta}(\gamma)}{d \gamma}\right\}=\frac{3 \bar{c}_{\theta}}{1+\bar{c}_{\theta}}
$$

Note that the second equation in Eq. (21) can also be obtained from Eq. (19). The variation of the correction factor $c_{\beta}$ against $\gamma$ is shown in Fig. 2. The thick dashed line in Fig. 2 shows the trend of the correction factor $c_{\beta}$ against $\gamma$, tending to the correct gradients when $\gamma$ tends to infinity or to zero, and passing through $c_{\beta}(1)=1.6$. From Fig. 2, the following approximate assumption can be made:

$$
\left(\frac{d c_{\beta}}{d \gamma}\right)_{\gamma \rightarrow 0} \approx \frac{c_{\beta}(1)}{1}
$$

Therefore, the approximate value of $\bar{c}_{\theta}$ is obtained as 


$$
\bar{c}_{\theta} \approx \frac{8}{7}
$$

which will be examined in Section 3. Also in Section 3, an even more accurate value for $\bar{c}_{\theta}$ is found to be $6 / 5$. The variation of the correction factor $c_{\theta}(\gamma)$ can then be expressed as

$$
c_{\theta}(\gamma)=\bar{c}_{\theta}^{\left[\hat{c}_{\beta}^{1 / 2}(1)-\hat{c}_{\beta}^{1 / 2}(\gamma)\right]}
$$

Eq. (24) is a choice obtained by inspection because when $\gamma=1, c_{\theta}=1$ is recovered, and when $\gamma \rightarrow \infty$ or $\gamma \rightarrow 0, c_{\theta}=\bar{c}_{\theta}$ is recovered. Using Eq. (24) and Eq. (17), the pure mode I $\theta_{1-2 D}$ mode is obtained. Then $\theta_{2-2 D}, \beta_{1-2 D}$ and $\beta_{2-2 D}$ are determined using the orthogonal property, that is,

$$
\begin{aligned}
& \theta_{2-2 D}(\gamma)=\operatorname{orthogonal}\left(\beta_{1-2 D}\right)=\operatorname{orthogonal}\left(\beta_{2-2 D}\right) \\
& \beta_{1-2 D}(\gamma,)=\operatorname{orthogonal}\left(\theta_{1-2 D}\right)=\operatorname{orthogonal}\left(\theta_{2-2 D}\right) \\
& \beta_{2-2 D}(\gamma)=\operatorname{orthogonal}\left(\theta_{1-2 D}\right)=\operatorname{orthogonal}\left(\theta_{2-2 D}\right)
\end{aligned}
$$

\section{Comparisons with Suo and Hutchinson's partition theory [1]}

In Fig. 3, a laminated unidirectional composite DCB with $M_{1 B}=1$ alone is considered, i.e. $M_{2 B} / M_{1 B}=0$. Note that for the purposes of this section, units are not required since only the ratios of geometrical dimensions and loads are of interest. The thickness ratio $\gamma=h_{2} / h_{1}$ varies from 100 to 0.01. Six partition theories (or 'rules') are compared. They are Suo and Hutchinson's theory [1], the authors' approximate rules 1 and 2 which are described in Refs. [2-4,6] and Ref. [4] respectively, the present theory with $\bar{c}_{\theta}=8 / 7$ and $\bar{c}_{\theta}=6 / 5$, and Luo and Tong's theory [7]. It is seen that the present theory with $\bar{c}_{\theta}=8 / 7$ is almost identical to Suo and Hutchinson's theory [1] when $0.1 \leq \gamma \leq 10$ and is slightly different when $\gamma<0.1$ or $\gamma>10$. The present theory with $\bar{c}_{\theta}=6 / 5$ is almost identical to Suo and Hutchinson's theory [1] over the entire range of $\gamma$ under consideration, i.e. $0.01 \leq \gamma \leq 100$, which represents most engineering applications. The other theories are generally approximate.

In Fig. 4, the same five partition theories as in Fig. 3 are compared against Suo and Hutchinson's theory [1] for a laminated unidirectional composite DCB with a variable $\gamma$ ratio, i.e. $0.01 \leq \gamma \leq 100$ and a variable $M_{2 B} / M_{1 B}$ ratio from -20 to 20 with $M_{1 B}=1$. These are the 
only two parameters that affect the partition $G_{I} / G$. The error is calculated as the difference between $G_{I} / G$ from the respective theory and $G_{I} / G$ from Suo and Hutchinson's theory [1]. The maximum error in Fig. 4 is capped at 0.05 in order to make a clearer comparison. Note that only Fig 4a has an error greater than 0.05; its maximum error is 0.10. Note that Fig. 3 represents a section through Fig. 4 at $M_{2 B} / M_{1 B}=0$. Again, the present theory with $\bar{c}_{\theta}=6 / 5$ is almost identical to Suo and Hutchinson's theory [1] over the entire range of $\gamma$ and $M_{2 B} / M_{1 B}$ under consideration. The present theory with $\bar{c}_{\theta}=8 / 7$ is almost identical to Suo and Hutchinson's theory [1] when $0.1 \leq \gamma \leq 10$ and is slightly different when $\gamma<0.1$ or $\gamma>10$. The other theories are generally approximate.

In Fig. 5, the same five partition theories are compared against Suo and Hutchinson's theory [1] for a laminated unidirectional composite DCB, where axial forces have been included. Again, the thickness ratio $\gamma$ varies from 100 to 0.01 . The loading $N_{1 B} / M_{1 B}$ ratio varies from -20 to 20 with $M_{1 B}=1$ and $N_{2 B}=M_{2 B}=0$. As for Fig. 4, the maximum error in Fig. 5 is also capped at 0.05 in order to make a clearer comparison. Note that only Fig 5a has an error greater than 0.05; its maximum error is 0.16 . Again, the present theory with $\bar{c}_{\theta}=6 / 5$ is almost identical to Suo and Hutchinson's theory [1] over the entire range of $\gamma$ and $N_{1 B} / M_{1 B}$ under consideration. The present theory with $\bar{c}_{\theta}=8 / 7$ is almost identical to Suo and Hutchinson's theory [1] when $0.1 \leq \gamma \leq 10$ and is slightly different when $\gamma<0.1$ or $\gamma>10$. The other theories are generally approximate.

\section{Conclusions}

A powerful method for partitioning mixed-mode fractures has been developed for rigid interface fractures in laminated unidirectional DCBs by taking 2D elasticity into consideration in a novel way. The new partition theory agrees very well with Suo and Hutchinson's partition theory [1].

This novel method will overcome the limitations of the conventional 2D-elasticity-based partition theory [1] when dealing with more complex problems, for example, in the bimaterial case where the partition relies on extensively tabulated numerical results over a finite range of geometries and material configurations [1]. The present method is currently being extended to bimaterial DCBs and the results will be reported in the near future. 


\section{References}

[1] Suo Z, Hutchinson JW. Interface crack between two elastic layers. International Journal of Fracture Mechanics 1990;43:1-18.

[2] Wang S, Harvey C. A theory of one-dimensional fracture. Composite Structures 2012;94:758-767. Also a plenary lecture at the $16^{\text {th }}$ International Conference on Composite Structures (ICCS16), 28-30 June 2011, Porto, Portugal.

[3] Wang S, Harvey C. Mixed mode partition theories for one dimensional fracture. Engineering Fracture Mechanics 2012;79:329-352. Also a plenary lecture at the 8th International Conference on Fracture and Strength of Solids (FEOFS 2010), 7-9 June 2010, Kuala Lumpur, Malaysia.

[4] Harvey CM, Wang S. Mixed-mode partition theories for one-dimensional delamination in laminated composite beams. Engineering Fracture Mechanics 2012;96:737-759.

[5] Wang S, Harvey CM, Guan L. Partition of mixed modes in layered isotropic double cantilever beams with non-rigid cohesive interfaces. Engineering Fracture Mechanics 2013;111:1-25.

[6] Harvey CM, Wang S. Experimental assessment of mixed-mode partition theories. Composite Structures 2012;94:2057-2067.

[7] Luo Q, Tong L. Analytical formulas of energy release rates for delamination using globallocal method. International Journal of Solids and Structures 2012;49:3335-3344.

\section{Appendix: The coefficient matrix $[C]$ of the ERR $G$ in Eq. (1)}

$$
[C]=\frac{1}{\bar{E} b^{2} h_{1}^{3} \gamma^{3}(1+\gamma)^{3}}\left[\begin{array}{ccc}
6 \gamma^{4}\left(\gamma^{2}+3 \gamma+3\right) & -6 \gamma^{3} & 3 h_{1} \gamma^{1} \\
-6 \gamma^{3} & 6\left(3 \gamma^{2}+3 \gamma+1\right) & 3 h_{1} \gamma^{4} \\
3 h_{1} \gamma^{4} & 3 h_{1} \gamma^{4} & h_{1}^{2} \gamma^{4}\left(\gamma^{2}-\gamma+1\right) / 2
\end{array}\right]
$$




\section{Figure list}

Fig. 1: A laminated unidirectional composite DCB. (a) General description. (b) Details of the $\Delta a$-length crack influence region.

Fig. 2: Variation of the correction factor $c_{\beta}(\gamma)$ with $\gamma$.

Fig. 3: Comparisons between various partition theories when $M_{1 B}=1$ and $N_{1 B}=N_{2 B}=M_{2 B}=0$.

Fig. 4: Magnitude of $G_{I} / G$ error of various partition theories relative to Suo and Hutchinson's theory [1] with $0.01 \leq \gamma \leq 100,-20 \leq M_{2 B} / M_{1 B} \leq 20, N_{1 B}=N_{2 B}=0, M_{1 B}=1$.

Fig. 5: Magnitude of $G_{I} / G$ error of various partition theories relative to Suo and Hutchinson's theory [1] with $0.01 \leq \gamma \leq 100,-20 \leq N_{1 B} / M_{1 B} \leq 20, N_{2 B}=M_{2 B}=0, M_{1 B}=1$. 


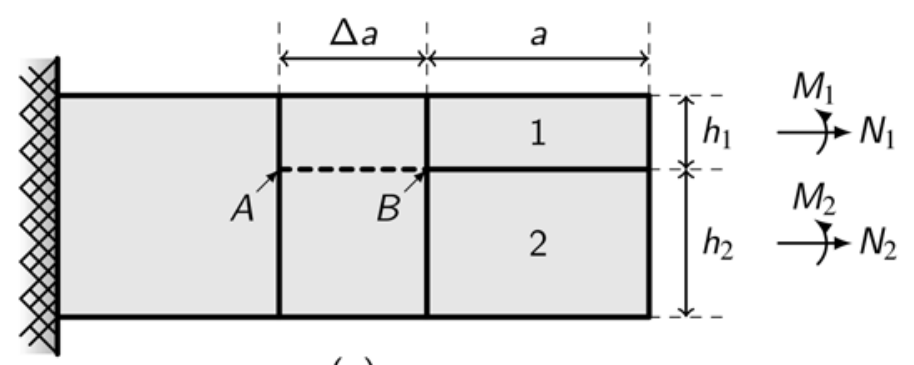

(a)

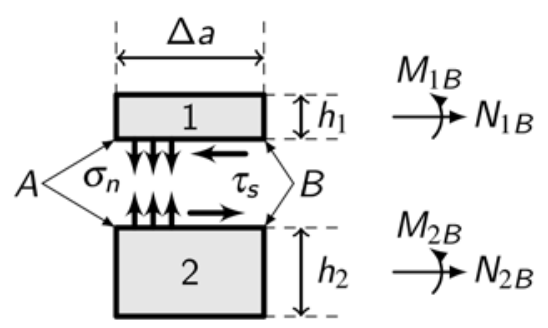

(b)

Fig. 1: A laminated unidirectional composite DCB. (a) General description. (b) Details of the $\Delta a$-length crack influence region. 


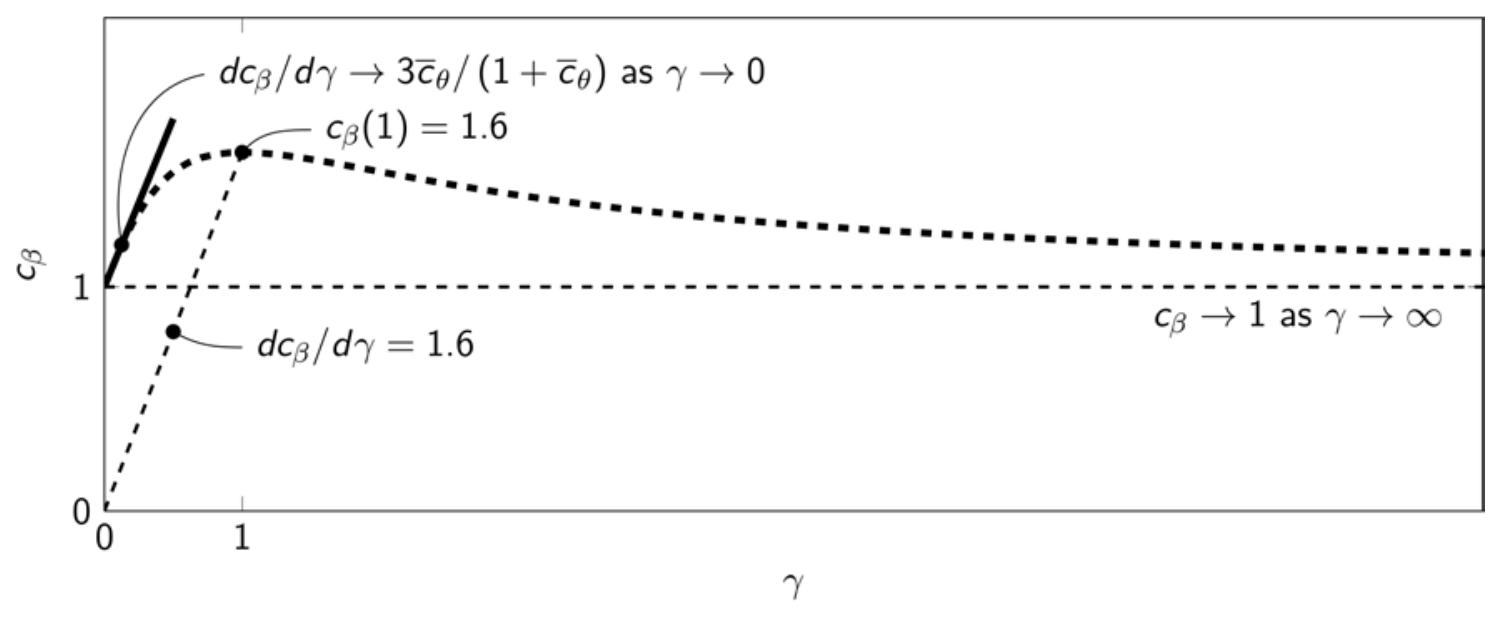

Fig. 2: Variation of the correction factor $c_{\beta}(\gamma)$ with $\gamma$. 


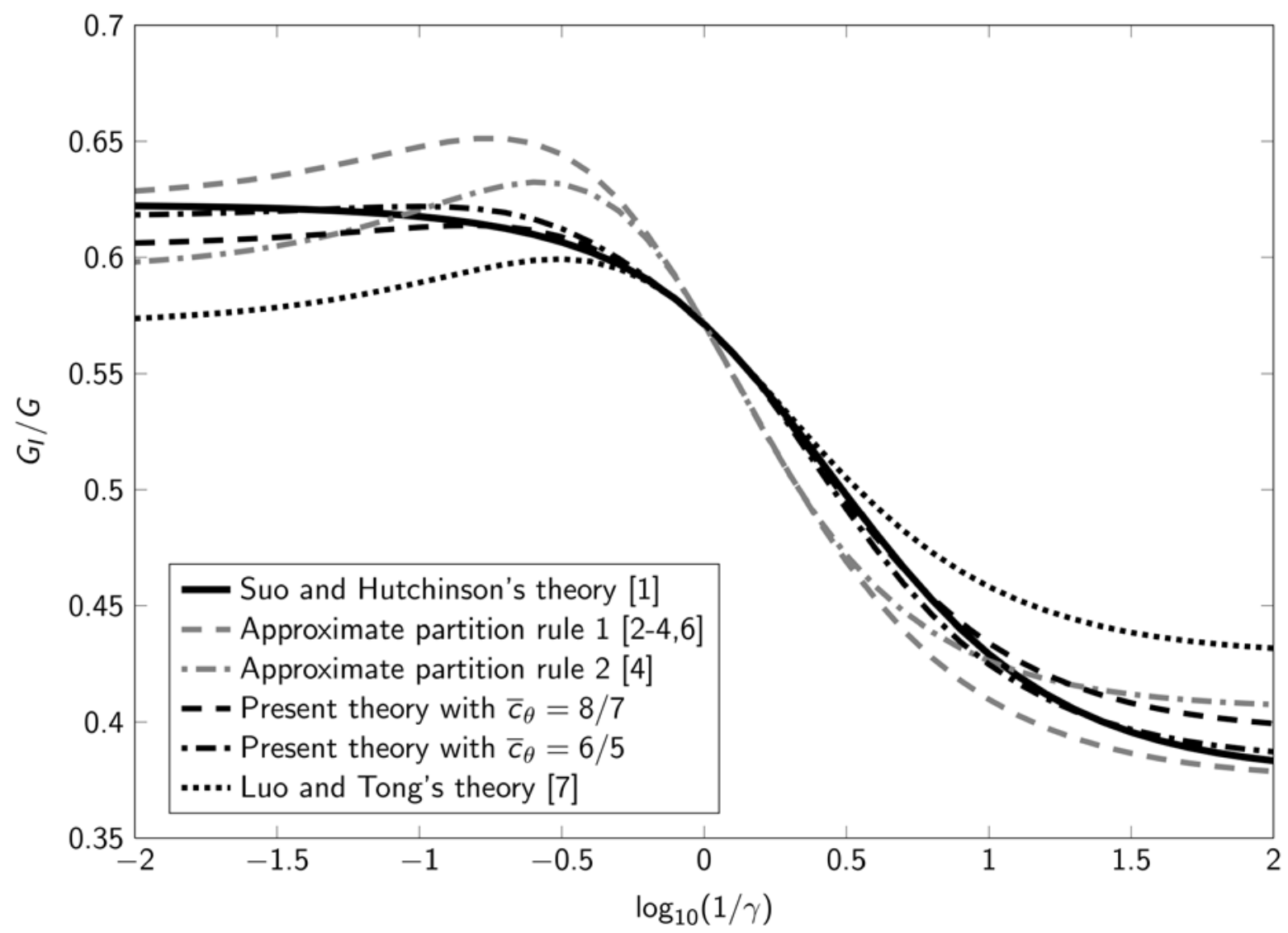

Fig. 3: Comparisons between various partition theories when $M_{1 B}=1$ and $N_{1 B}=N_{2 B}=M_{2 B}=0$. 


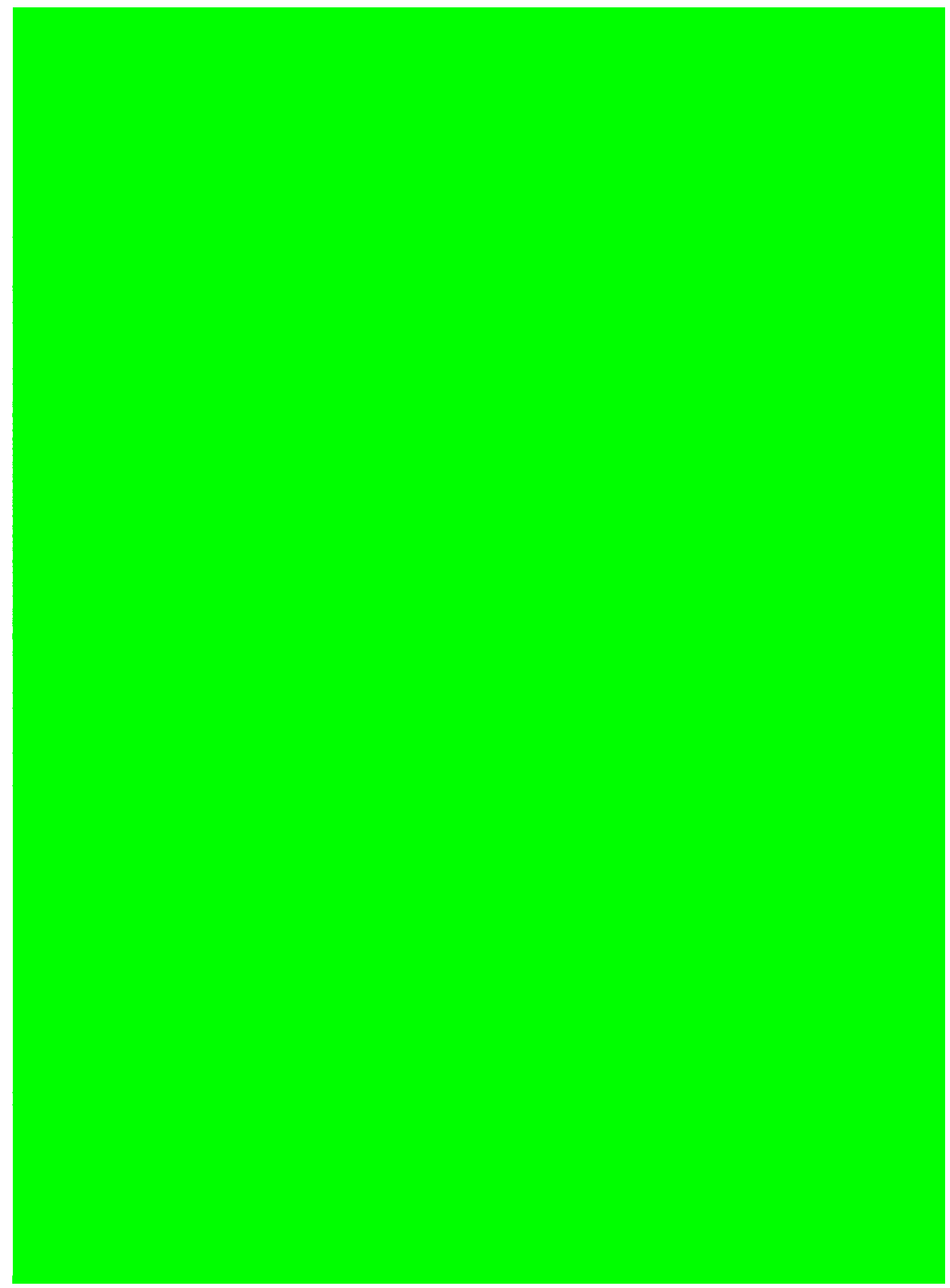

Fig. 4: Magnitude of $G_{I} / G$ error of various partition theories relative to Suo and Hutchinson's theory [1] with $0.01 \leq \gamma \leq 100,-20 \leq M_{2 B} / M_{1 B} \leq 20, N_{1 B}=N_{2 B}=0, M_{1 B}=1$. 
Fig. 5: Magnitude of $G_{I} / G$ error of various partition theories relative to Suo and Hutchinson's theory [1] with $0.01 \leq \gamma \leq 100,-20 \leq N_{1 B} / M_{1 B} \leq 20, N_{2 B}=M_{2 B}=0, M_{1 B}=1$. 